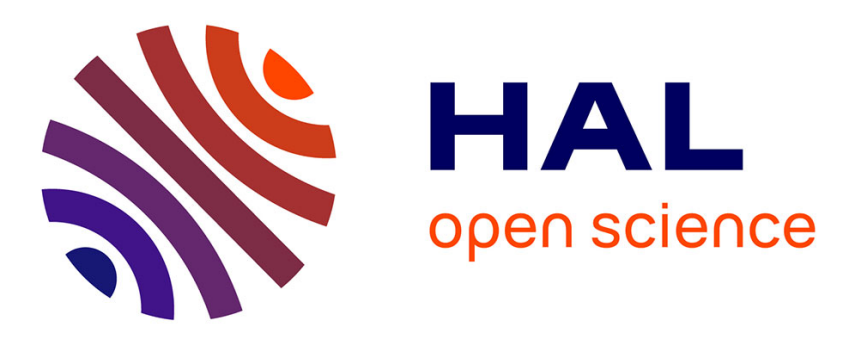

\title{
Strain resolution of scanning electron microscopy based Kossel microdiffraction
}

Denis Bouscaud, Adam Morawiec, Raphaël Pesci, Sophie Berveiller, Etienne Patoor

\section{- To cite this version:}

Denis Bouscaud, Adam Morawiec, Raphaël Pesci, Sophie Berveiller, Etienne Patoor. Strain resolution of scanning electron microscopy based Kossel microdiffraction. Journal of Applied Crystallography, 2014, 47 (5), pp.1699-1707. 10.1107/S1600576714019402 . hal-01110070

\section{HAL Id: hal-01110070 \\ https://hal.science/hal-01110070}

Submitted on 27 Jan 2015

HAL is a multi-disciplinary open access archive for the deposit and dissemination of scientific research documents, whether they are published or not. The documents may come from teaching and research institutions in France or abroad, or from public or private research centers.
L'archive ouverte pluridisciplinaire HAL, est destinée au dépôt et à la diffusion de documents scientifiques de niveau recherche, publiés ou non, émanant des établissements d'enseignement et de recherche français ou étrangers, des laboratoires publics ou privés. 


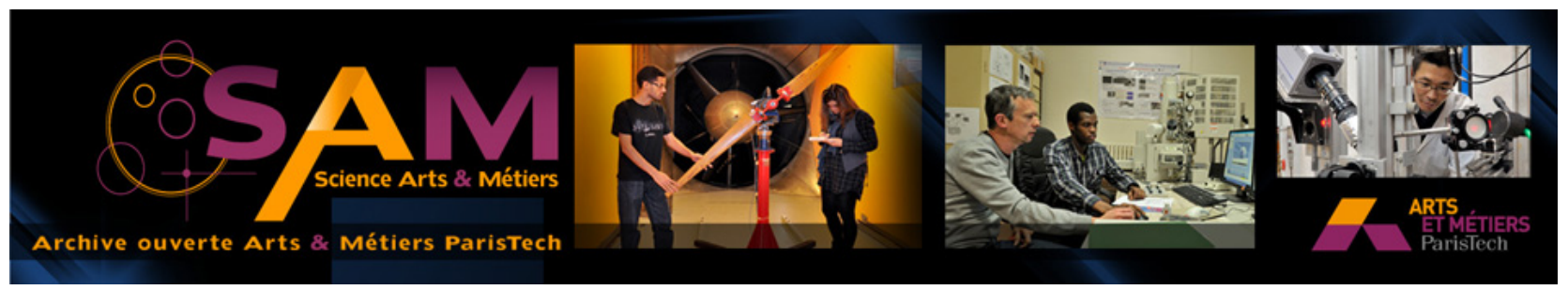

Science Arts \& Métiers (SAM)

is an open access repository that collects the work of Arts et Métiers ParisTech researchers and makes it freely available over the web where possible.

This is an author-deposited version published in: http://sam.ensam.eu

Handle ID: .http://hdl.handle.net/10985/9278

\section{To cite this version :}

Denis BOUSCAUD, Adam MORAWIEC, Raphaël PESCI, Sophie BERVEILLER, Etienne PATOOR - Strain resolution of scanning electron microscopy based Kossel microdiffraction Journal of Applied Crystallography - Vol. 47, n5, p.1699-1707 - 2014 


\title{
Strain resolution of scanning electron microscopy based Kossel microdiffraction
}

\author{
D. Bouscaud, ${ }^{\mathrm{a} *}$ A. Morawiec, ${ }^{\mathrm{b}}$ R. Pesci, ${ }^{\text {a }}$ S. Berveiller ${ }^{\mathrm{a}}$ and E. Patoor ${ }^{\mathrm{a}}$ \\ aLEM3 UMR CNRS 7239, Arts et Métiers ParisTech, 4 rue Augustin Fresnel, F-57078 Metz, France, \\ and ${ }^{\mathbf{b}}$ Institute of Metallurgy and Materials Science, Polish Academy of Sciences, Reymonta 25, PL- \\ 30-059 Kraków, Poland. Correspondence e-mail: denis.bouscaud@ensam.eu
}

\begin{abstract}
Kossel microdiffraction in a scanning electron microscope enables determination of local elastic strains. With Kossel patterns recorded by a CCD camera and some automation of the strain determination process, this technique may become a convenient tool for analysis of strains. As for all strain determination methods, critical for the applicability of the Kossel technique is its strain resolution. The resolution was estimated in a number of ways: from the simplest tests based on simulated patterns (of an Ni alloy), through analysis of sharp experimental patterns of Ge, to estimates obtained by in situ tensile straining of single crystals of the Ni-based superalloy. In the latter case, the results were compared with those of conventional X-ray diffraction and synchrotron-based Kossel diffraction. In the case of high-quality Ge patterns, a resolution of $1 \times$ $10^{-4}$ was reached for all strain tensor components; this corresponds to a stress of about $10 \mathrm{MPa}$. With relatively diffuse patterns from the strained Ni-based superalloy, under the assumption of plane stress, the strain and stress resolutions were $3 \times 10^{-4}$ and $60 \mathrm{MPa}$, respectively. Experimental and computational conditions for achieving these resolutions are described. The study shows potential perspectives and limits of the applicability of semiautomatic Kossel microdiffraction as a method of local strain determination.
\end{abstract}

\section{Introduction}

Understanding local stresses in crystalline materials is of importance for the comprehension of material properties and for selecting optimal processing conditions. Various techniques are used to determine such stresses. Most measurements are performed at the macroscopic scale (Ruud, 1982). However, in the presence of local microstructural inhomogeneities, stress heterogeneities arise at the microscopic scale (Barbe et al., 2001). The microstructure of most engineering materials is made up of distinct crystallites having specific sizes, shapes, crystallographic orientations and neighborhoods. When crystallites are deformed as part of an aggregate, their anisotropy gives rise to strain incompatibilities. As a result, second- and third-order stresses appear: the stress state in each crystallite is distinct, as it depends on the crystallite's ability to accommodate the deformation imposed by the aggregate. Furthermore, stress heterogeneities are introduced by grain boundaries and the surface.

To measure representative local stress states in polycrystalline materials with typical grain sizes $(1-100 \mu \mathrm{m})$, adequate methods need to be applied. There are two crucial factors determining the quality of a technique of local stress measurement. These are the spatial resolution and the stress (or strain) resolution. High spatial resolution of the order of a few micrometres or better is indispensable for investigating stresses and stress gradients inside individual crystallites. Also, the accuracy of strain/stress determination is of critical importance. In most applications, strain variations of about one part per ten thousand are of interest, and consequently, only methods providing at least this level of accuracy are of use. Stress states can be estimated via numerical models, but experimental data are still needed as reference and for verification of the models.

Several experimental techniques are available to study local stress states. Laue diffraction using a microfocused synchrotron beam is being developed in a number of beamlines around the world (e.g. Tamura et al., 2005), but access to this technique is limited. Micro-Raman spectroscopy is a powerful tool for the analysis of semiconductors (e.g. De Wolf et al., 1999), but it relies on assumptions about strain state, and it is not applicable to metallic samples. Convergent beam electron diffraction in a transmission electron microscope allows for strain measurements at a submicrometre scale (e.g. Krämer et $a l .$, 2000), but since thin foils are used, the issue of stress relaxation arises (Clément et al., 2004). Another technique, which seems to be more accessible, is high-resolution electron backscatter diffraction (EBSD), but only the third-order stresses can be deduced from EBSD patterns (e.g. Wilkinson $e t$ al., 2009).

In this situation, it is worth considering local stress determination using Kossel microdiffraction. Kossel interference was first observed in 1934 (Kossel et al., 1935). In the 1960s, 
with the use of electron probe microanalysers, the Kossel technique was considerably advanced (Tixier \& Waché, 1970). Then, it was adapted to scanning electron microscopy (SEM) (Dingley, 1975); this enables the recording of diffraction patterns while the microstructure of the specimen is observed. Nowadays, with the application of digital cameras for pattern acquisition (Pesci et al., 2006; Böhling \& Bauch, 2007), the analysis of SEM-based Kossel patterns can be automated to a considerable extent. For a review of Kossel diffraction and its applications, see Lider (2011).

There are various claims on the spatial resolution of the SEM-based Kossel technique (Dingley, 1975; Böhling \& Bauch, 2007). It is believed to be in the broad range from 1 to $10 \mu \mathrm{m}$. Therefore, the technique can be used for investigating elastic strains inside sufficiently large individual crystallites, but there is an essential question about the levels of strain that can be measured. Strain determination is linked to lattice parameter determination. Taking this into account, various sources on the accuracy of the Kossel technique can be quoted (Tixier \& Waché, 1970; Dingley, 1975; Lamaze \& Despujols, 1976; Cunningham \& Ashbee, 1990; Böhling \& Bauch, 2007; Langer \& Daebritz, 2010; Lider, 2011), and the estimates of strain resolution vary from $5 \times 10^{-4}$ to $1 \times 10^{-6}$. However, most of the strain or lattice parameter determination methods require special crystal orientations or particular configurations of Kossel lines. These requirements are not satisfied when grains in polycrystalline materials are investigated. Moreover, for stresses measured in individual crystallites, one must take into account the crystal anisotropy, i.e. multiple components of stress tensors need to be determined.

The purpose of the present paper is to estimate the strain resolution in the case of SEM-generated digitally recorded Kossel patterns originating from crystals of arbitrary orientation. Such patterns are routinely collected at LEM3 from various materials, but here the focus will be just on results related to the strain resolution. First, the experimental setup for recording Kossel patterns in a scanning microscope and the method for acquiring the strain and the stress tensors are briefly described. Then, limits of the strain resolution of the technique are estimated by analyzing simulated patterns for a nickel alloy and high-quality experimental patterns of germanium. Finally, results for loaded crystals of the nickelbased alloy analyzed in situ by SEM are presented. The results are compared with similar data obtained from Kossel patterns generated by a microfocused synchrotron beam and with data from classical X-ray diffraction. These measurements demonstrate the applicability of the Kossel technique to cases of practical importance. The study also shows the limits of semiautomatic SEM-based Kossel microdiffraction as a method of strain determination; these limits are crucial for decisions about investing in the development of automatic Kossel-based strain determination systems.

\section{Recording Kossel patterns using SEM}

The Kossel patterns are produced in a scanning electron microscope and captured by an X-ray-sensitive two-dimen- sional detector. Fig. 1 shows the experimental setup for the acquisition of Kossel patterns. A focused electron beam excites atoms and causes X-ray emission. Within the geometric description of X-ray diffraction, a stack of crystallographic planes with interplanar spacing exceeding half of the wavelength of the X-ray radiation leads to a Kossel cone with the apex at the point where the electron beam hits the specimen. The cone axis coincides with the normal to the diffracting planes, and the semi-apex angle of the cone equals $90^{\circ}$ minus the Bragg angle. A Kossel pattern consists of a set of conics: intersections of the Kossel cones with a planar detector. The $\mathrm{X}$-ray interference originating from the crystal generates light on a fluorescent screen. Patterns are recorded by a CCD coupled to the screen through a fiber-optic taper. Digitally recorded patterns can be directly analyzed by appropriate software.

The scanning microscope used in this study was a JEOL 5800 with a thermionic emission tungsten filament operating at a voltage of $30 \mathrm{kV}$. It was equipped with an 11 megapixel highresolution 12-bit Peltier-cooled CCD camera ('VHR-11', Photonic Science Ltd) with an optical pixel size of $9 \mu \mathrm{m}$. Tensile tests were carried out directly in the microscope using a $5 \mathrm{kN}$ tensile/compression module (MicroMecha).

Kossel patterns have a low signal-to-background ratio (Bauch et al., 2000). In order to obtain patterns with sufficiently strong contrast, multiple frames (five to 20) are blended into one by averaging pixel-to-pixel intensities. Then, the background (collected in the scanning mode) is subtracted. The acquisition time depends very much on the required quality of the pattern and the microscope operating conditions. For the patterns used below, 20 frames were collected to be blended into a single pattern, and the acquisition time per frame was $10-30 \mathrm{~s}$. The time per frame is selected on the basis of a gray-level histogram; the point is to have the widest distribution possible without saturating the detector. The number of frames is selected by observing line profiles; the

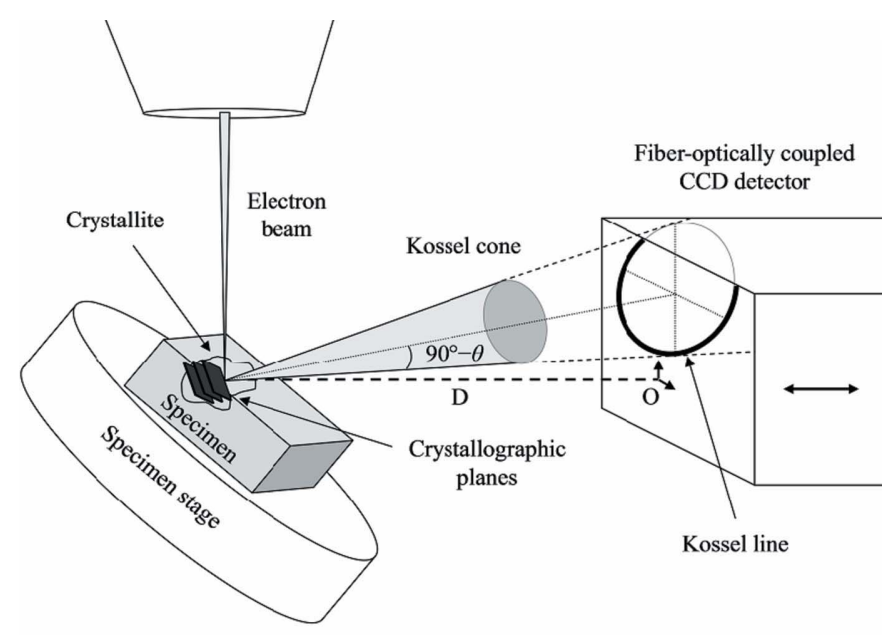

Figure 1

SEM chamber

Schematic of the Kossel microdiffraction experimental setup. D is the specimen-to-detector distance; $\mathrm{O}$ marks the pattern center and $\theta$ denotes the Bragg angle. 
goal is to have an optimal signal-to-noise ratio. The ratio is improved by increasing the number of frames, but it can deteriorate with increasing time per frame because of thermal noise in the camera. The acquisition time depends on the sample-to-detector distance. The camera body is supported by a powered linear stage which allows for adjusting the distance during an imaging session while maintaining vacuum. With decreasing distance, the exposure time also decreases, and concurrently, the acceptance angle and the number of conics intercepted by the detector increase. Therefore, it is advantageous to have a small sample-to-detector distance and a large field of view. The distance used in this study was about $40 \mathrm{~mm}$, and the field of view of the camera on the fluorescent screen was $36 \times 24 \mathrm{~mm}$.

In Kossel microdiffraction, X-rays are emitted isotropically, and therefore Kossel patterns can be obtained regardless of the magnitudes of specimen tilt and working distance. These two parameters can be chosen to have the most curved Kossel lines in the diffraction pattern; the higher the Bragg angle, the better the strain sensitivity. However, when the tensile stage is used, the margins for specimen movements become small. In this case, a working distance of about $20 \mathrm{~mm}$ and a tilt angle of $40^{\circ}$ were used.

The Kossel technique requires relatively high probe current, comparable to that of wavelength-dispersive X-ray spectroscopy. This causes a local rise of temperature of the investigated material and thus influences the lattice parameters. A previous study showed that in conductive materials the temperature rise is too small to affect the strain measurement (Bouscaud et al., 2012).

The camera was run in distortion correction mode, with optical distortions in the image removed using a custom map file containing the per-pixel correction data specific to the particular camera. The accuracy of a similar approach was estimated to be greater than 0.25 pixels (Barna et al., 1999). Before final analysis, the recorded patterns were consolidated using $4 \times 4$ binning. Thus, the optical distortions in the analyzed (consolidated) patterns are expected to be smaller than $0.25 / 4$ pixels.

\section{Analysis of experimental Kossel patterns}

The recorded Kossel patterns were analyzed using a dedicated software package (KSLStrain; Morawiec et al., 2008; a demo version of the program can be downloaded from http:// imim.pl/personal/adam.morawiec/A_Morawiec_Web_Page/ downloads.html). The input data are the microscope settings (the sample tilt angle, the detector pixel size, approximate location of the pattern center and approximate sample-todetector distance) and the material properties (crystal structure and X-ray wavelength). The main task of the operator is to provide locations of Kossel lines, and this needs to be done with the highest possible precision. The Kossel lines are marked manually using a computer mouse. The intensity profile in the direction perpendicular to the Kossel line (averaged over a short segment of the line) is used to refine positions of the markers with a resolution of $1 / 10$ of the pixel size (Fig. 2). For every marker, the best position is chosen according to the rules described below. The software also calculates deviations of marked points from the corresponding ideal conic lines. This check of the data consistency is an important tool for improving the accuracy of marker locations; imprecisely located markers are identified by large deviations, and their locations can be corrected.

Kossel lines exhibit various intensity profiles: they are bright or dark depending on the line intensity with respect to the background. A profile can also be more complex, with bright and dark parts ('helldunkel Struktur'). Fig. 3 shows the three types of profiles. The two maxima noticeable on the bright profile of Fig. $3(a)$ are due to the $K \alpha_{1}-K \alpha_{2}$ splitting clearly visible for high $h k l$ reflections.

Precise determination of the locations of Kossel lines is a critical point of the strain analysis. The difficulty lies in the positioning of the marking points. The maximum of the intensity profile does not always fit the location given by the geometric theory of diffraction. In order to deal with the complex line profiles, the following guidelines for marking the conics have been developed: In the case of bright lines, marking points are located at the maximum intensity of the $K \alpha_{1}$ reflection (i.e. on the convex side of the conic). Since the contrast of (relatively rare) dark lines is poor, they are not used for strain determination (although they can be used for indexing of the patterns). In the case of bright-dark conics, marking points are located at the inflection points of the intensity profile. This particular location has been verified by comparing line profiles with simulated patterns obtained after having marked only bright lines. The above rules combined with a geometric description of diffraction are a substitute for a complicated exact simulation of pattern intensities.

Using the input parameters and locations of the markers, the Kossel lines are automatically indexed by the package KiKoCh (Morawiec, 1999) integrated into KSLStrain. The final stage consists in the refinement of lattice parameters and thus the determination of the full strain tensor $\varepsilon$. The refinement is based on an optimization procedure matching the marked experimental Kossel conics to corresponding conics in simulated patterns. The matching is performed using $K L E B S$, an optimization procedure minimizing deviations between marked locations on experimental lines and lines in kinematically simulated patterns. The objective function is based directly on the formal equation of the Kossel lines. The $K L E B S$ algorithm is described in detail by Morawiec (2007). The crystallite orientation, the location of the pattern center and the sample-to-detector distance are refined alongside the lattice parameters. The computation of the strain tensor is carried out in the crystal coordinate system. If needed, the full stress tensor $\sigma$ is calculated using Hooke's law, $\sigma=C \varepsilon$, where $C$ is the elastic stiffness tensor of the crystal. Knowing the crystallographic orientation and the specimen tilt, the components of the strain and stress tensors in the sample coordinate system are computed.

Unless a user imposes extra restrictions, the minimization involves 12 parameters (six strain components, three orientation parameters, two pattern center coordinates and the 


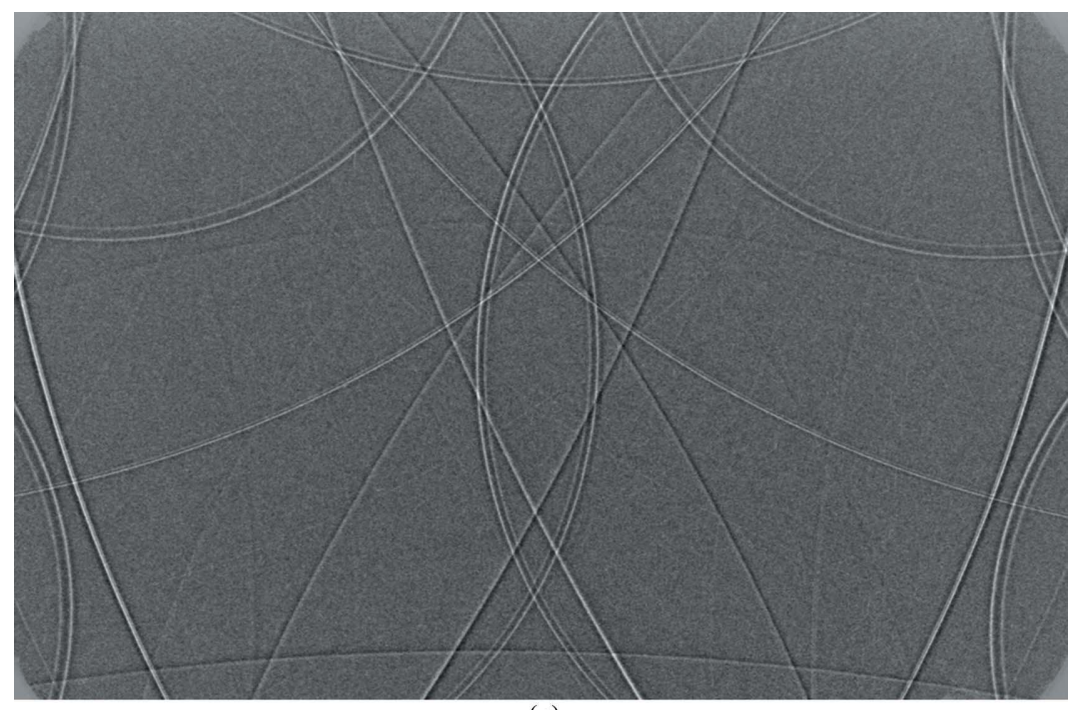

(a)

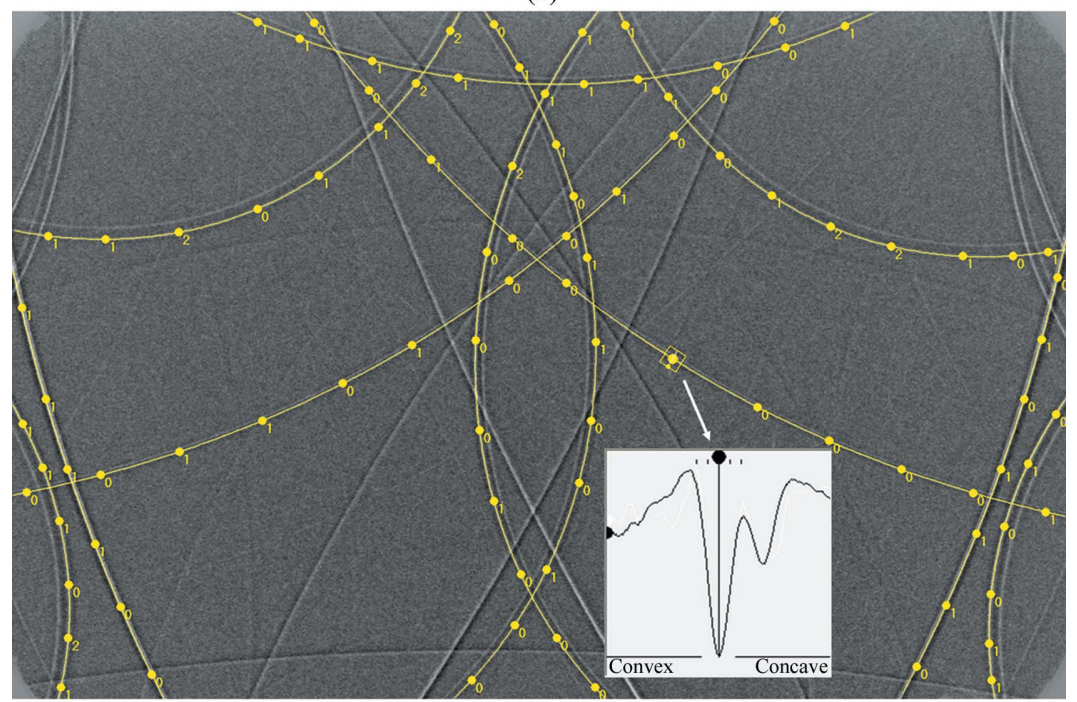

(b)

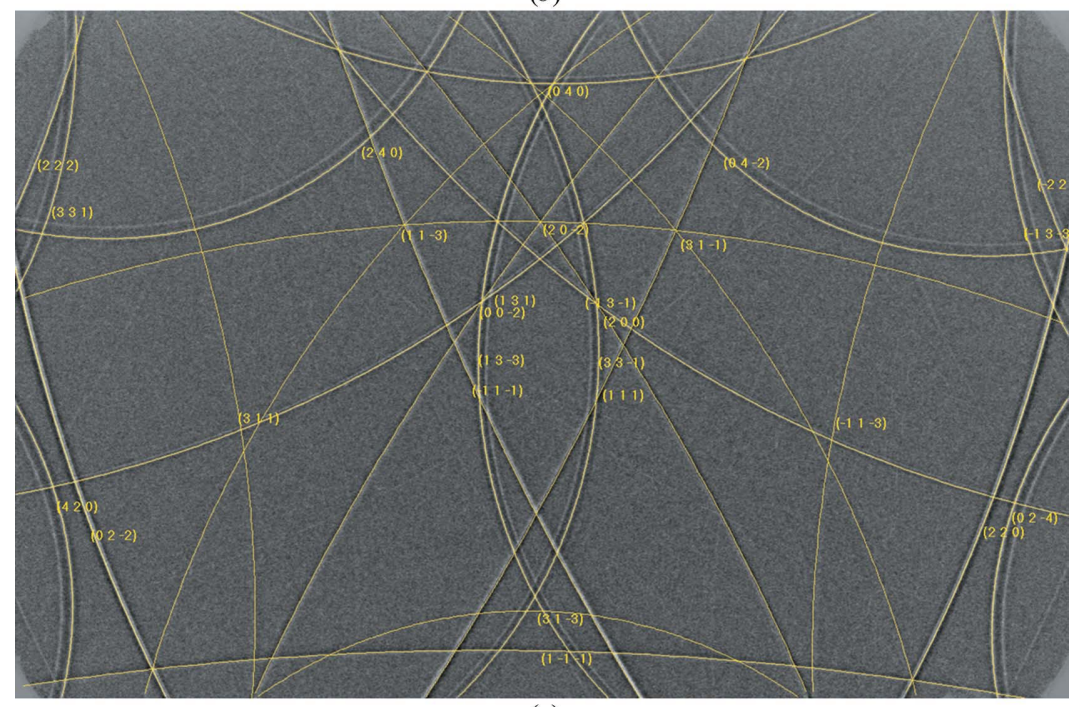

Figure 2

(c)

(a) Experimental Kossel pattern obtained from a copper crystal. (b) Conics marked on the pattern. The numbers represent magnitudes (in 1/10 of the pixel size) of the deviations of each marker from the corresponding ideal conic. Also, an intensity profile of a line at the indicated point is shown. (c) The corresponding simulated pattern after the strain calculation. sample-to-screen distance). The program shows one-dimensional sections through the objective function and outputs correlation coefficients between fitted parameters. In the considered cases, at the scale of $10^{-4}$, these sections did not exhibit any local minima. The particular values of the correlation coefficients depend on the case under study, but generally, most of the correlations between strain tensor components and other parameters are weak (with absolute values below 0.5); some are stronger, but they rarely exceed 0.7 . There is always a strong correlation between rotation about the $x$ axis ( $y$ axis) and the $y$ coordinate ( $x$ coordinate) of the pattern center. This is expected because the impact of a small rotation of a crystal is nearly the same as that of a small shift of the pattern center. This is of little importance because the pattern center and rotation parameters are auxiliary variables.

The KSLStrain software also allows for calculating the strain tensor components in the crystal coordinate system under the assumption of plane stress in the sample coordinate system (i.e. with $\sigma_{13}=\sigma_{23}=\sigma_{33}=0$, where the axis ' 3 ' is perpendicular to the free surface). If applicable, this assumption reduces the number of fitted parameters, and it improves the resolution when the number of conics in the pattern is small.

As the goal is to determine absolute stress tensors, the reference lattice parameters $a$ were obtained from the assumption that the stress in the direction normal to the free surface of the specimen is equal to zero $\left(\sigma_{33}=0\right)$ owing to the low X-ray penetration depth. This approach is standard when stresses are determined in polycrystalline materials using the $\sin ^{2} \psi$ method [see $\$ 2.11$ of the book by Hauk (1997)]. The same principle can be applied in analysis of single crystals (Ortner, 2005). The parameters obtained from X-ray diffraction (XRD) data were intended for XRD experiments and those from Kossel data for Kossel experiments, but actually the same values were found with the accuracy to the fourth significant digit, and these values were used. Such obtained reference parameters are also affected by errors, but since this is just one parameter per measurement, the errors are much smaller than those arising when multiple components of the strain tensor are fitted.

\section{Strain resolution}

There are several factors influencing the strain resolution. Assuming sufficient quality of the recorded patterns, the resolution depends on the 


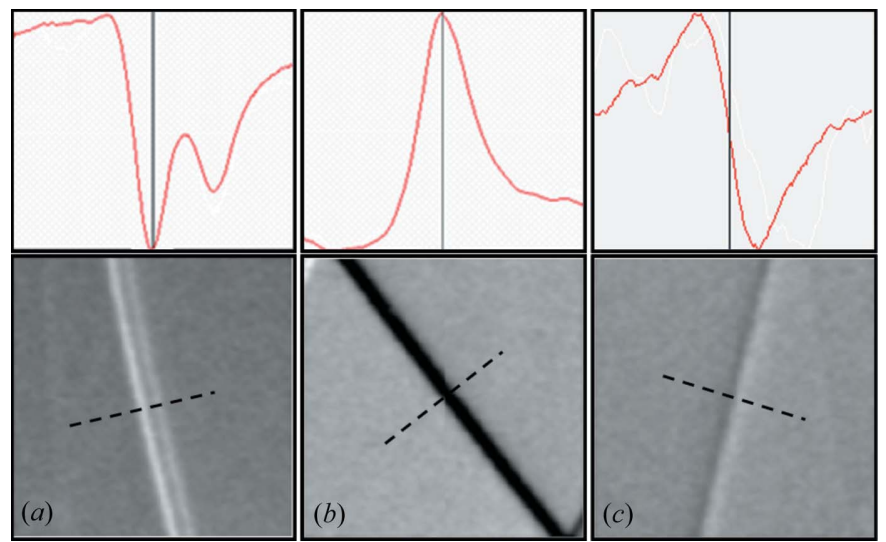

Figure 3

Typical intensity profiles of Kossel lines (averaged over 20 pixel long segments) and the corresponding (78 pixel wide) fragments of patterns from $\mathrm{Cu}$ : (a) bright, $(b)$ dark, $(c)$ bright-and-dark line.

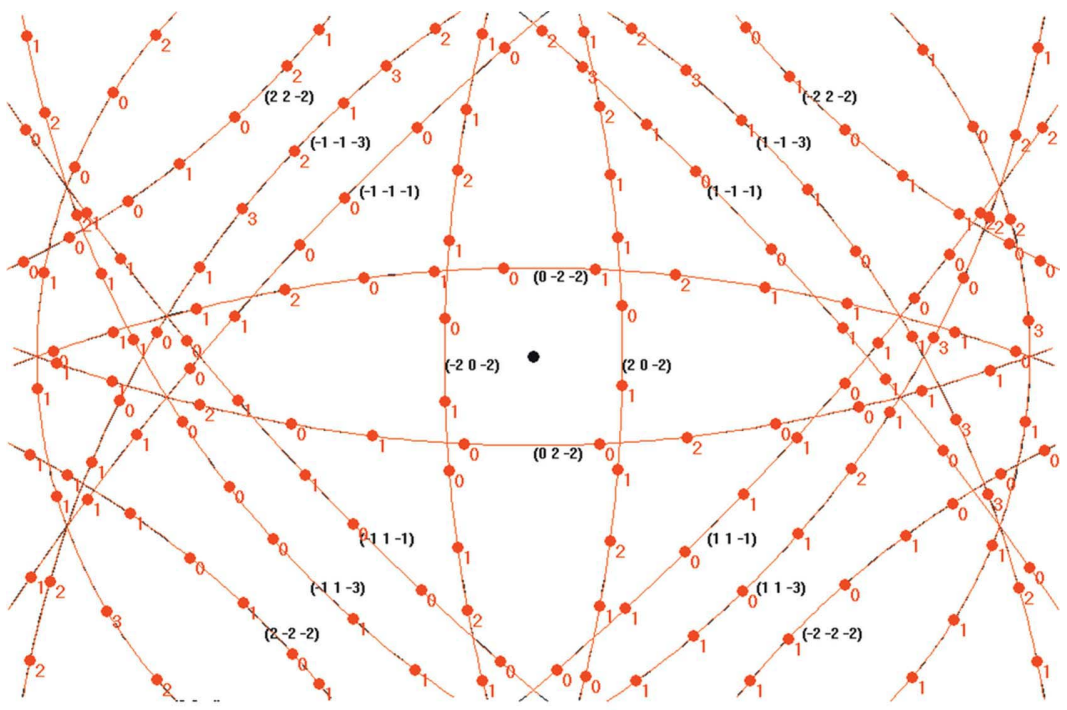

(a)

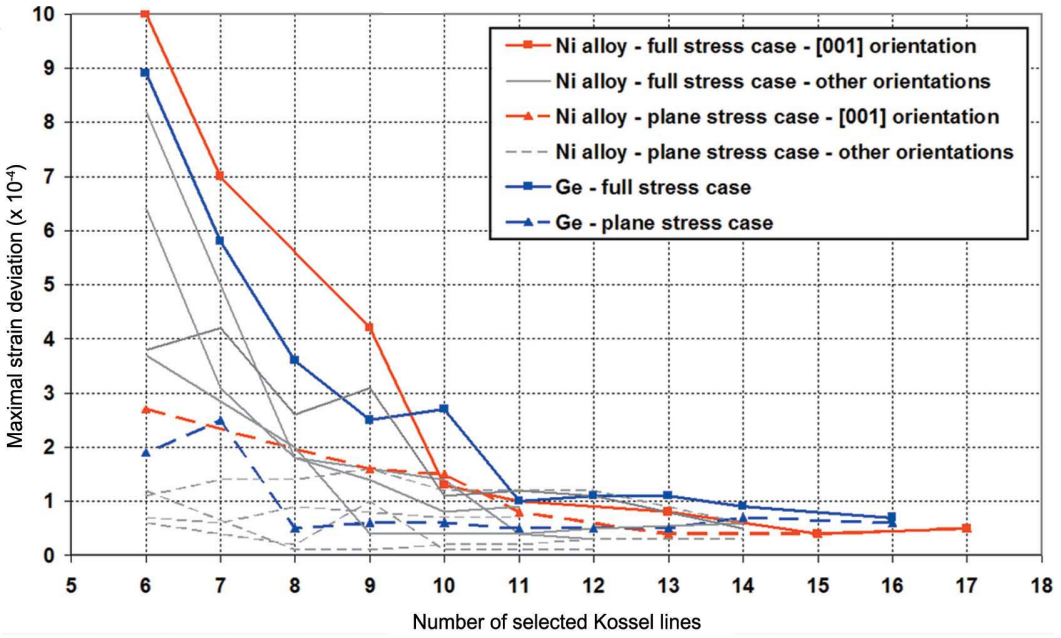

(b)

Figure 4

(a) Markers of conics superimposed on the simulated Kossel pattern of the Ni alloy; the locations of markers are used in the calculations. $(b)$ The largest strain deviation (in units of $10^{-4}$ ) after the refinement for five simulated $\mathrm{Ni}$ alloy patterns and for an experimental pattern of a Ge crystal versus the number of marked conics; both the full stress and the plane stress cases are shown. solid angle covered by the pattern, the number of lines used in calculations and the magnitudes of the corresponding Bragg angles. For a given material and experimental setup, only the latter factor can be controlled. The influence of the number of lines on strain resolution has not been examined previously. It is, however, an important aspect of the strain determination procedure. Its influence was checked using Kossel line patterns simulated from data for a nickel alloy ( $c f . \S 5)$. These results are supported by recovering known lattice parameters from a sharp experimental pattern of Ge.

\subsection{Strain resolution from simulated patterns}

The simplest test to estimate the achievable strain resolution is to simulate a diffraction pattern for some parameters and then attempt to recover these parameters through the analysis devised for experimental patterns. Such a test was carried out with diffraction patterns of an $\mathrm{Ni}$ alloy. The patterns were simulated using KSLStrain. The lattice constant $a$ was set at $3.595 \AA$, and the assumed microscope settings were as in the experimental setup. The patterns were analyzed according to the procedure described in $\$ 3$. For instance, for the pattern shown in Fig. 4(a) with the [001] direction perpendicular to the detector, the maximum of 17 Kossel lines were marked. In all considered patterns, the lines were one pixel wide. The strain components were repeatedly fitted on the basis of a decreasing number of marked conics. The low-index reflections having low strain sensitivity were removed first. The largest absolute value of the six strain components, i.e. the maximum deviation of the resulting lattice parameters from the data used in the simulation, is plotted as a function of the number of marked conics in Fig. 4(b).

By analyzing the ideal simulated case, one obtains limits of the strain resolution. In other words, when the above approach is applied to a real material, its accuracy cannot be better than the deviations shown in Fig. 4(b). The tests demonstrate that, when the full stress state is calculated for the considered material and experimental setup, at least 11 Kossel conics need to be taken into account to reach an accuracy of $2 \times 10^{-4}$. Under the plane stress assumption, the same accuracy limit corresponds to seven Kossel conics. With a very large number of conics, the accuracy of individual strain tensor components is bounded by $0.5 \times$ $10^{-4}$.

\subsection{Strain resolution from sharp experimental patterns}

More realistic estimates of strain resolution can be obtained by recovering known lattice 
parameters from an experimental pattern. With this approach, camera distortions, diffusivity of patterns and true line profiles are taken into account. To carry out such tests, a germanium single crystal was used. The specimen was cut out from a microelectronic wafer. The case is still in a sense ideal, because Kossel patterns of Ge contain numerous lines with relatively sharp peaks in the line profiles. In practice, the strain sensitivity can be better only for materials leading to high-Braggangle conics.

The Kossel patterns were recorded at a random location of the sample. In the analysis of the patterns, an effort was made to maximize the number of used Kossel lines, but only bright lines with a noticeable $K \alpha_{1}-K \alpha_{2}$ splitting were taken into account; the dark-and-bright lines were not used. In the particular pattern shown in Fig. 5, 16 conics were marked. The reference lattice parameter was $a=5.658 \AA$. The elastic stiffness components used for stress calculation are listed in Table 1. The obtained full strain tensor and the corresponding stress tensor, both in the sample coordinate system, were

$$
\varepsilon=\left(\begin{array}{ccc}
-7 & 1 & -4 \\
& 1 & 3 \\
& & 9
\end{array}\right) \times 10^{-5}
$$

and

$$
\sigma=\left(\begin{array}{ccc}
-21 & 0 & -5 \\
& -11 & 3 \\
& & 0
\end{array}\right) \mathrm{MPa}
$$

In accordance with the method of determining the reference lattice parameter, the resulting stress in the direction normal to the free surface of the specimen is equal to zero $\left(\sigma_{33}=0\right)$. Like in the case of the simulated patterns of the Ni alloy, the strain was repeatedly calculated on the basis of a decreasing number of marked conics. The results are plotted in Fig. 4(b).

For reference, the same sample was also analyzed by conventional XRD using a four-circle goniometer and the

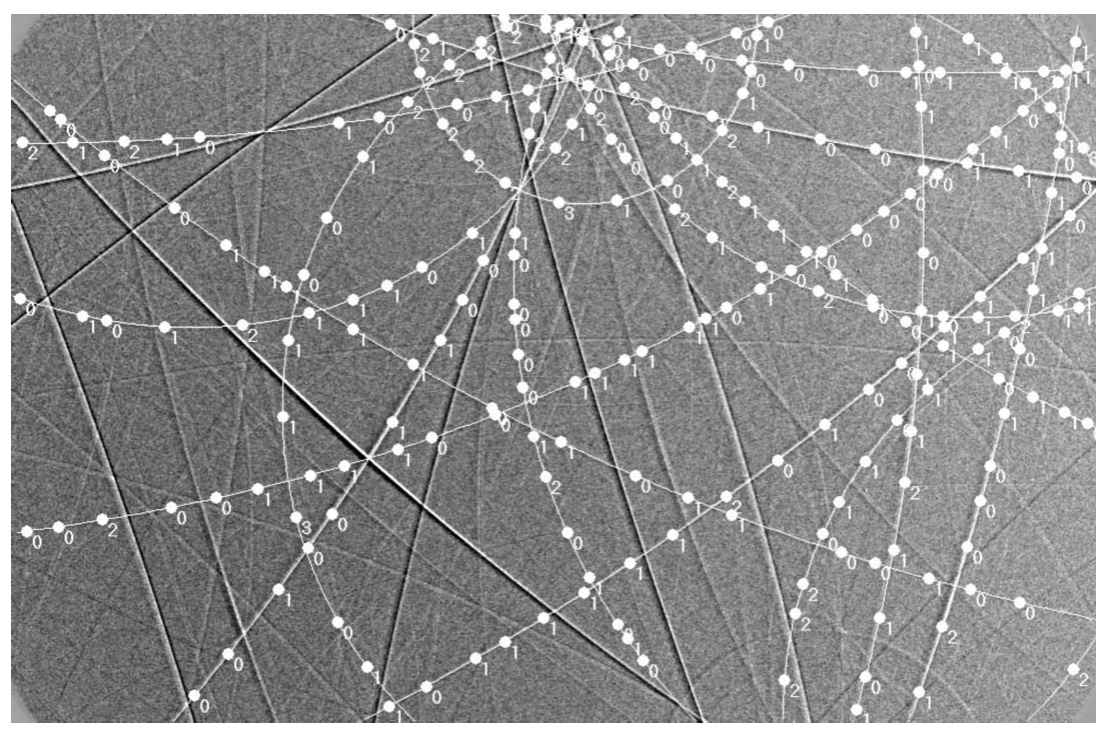

Figure 5

Experimental Kossel pattern obtained from the Ge crystal with manually marked conics.
Table 1

Components of the elastic stiffness tensor for Ge and the Ni superalloy.

\begin{tabular}{llcc}
\hline & $\mathrm{C}_{11}(\mathrm{GPa})$ & $\mathrm{C}_{12}(\mathrm{GPa})$ & $\mathrm{C}_{44}(\mathrm{GPa})$ \\
\hline $\mathrm{Ge}$ & 128.5 & 48.3 & 66.8 \\
Ni superalloy & 247 & 161 & 127 \\
\hline
\end{tabular}

method of Crostack et al. (1987). The full strain tensor and the corresponding stress tensor, both in the sample coordinate system, obtained by XRD were

$$
\varepsilon=\left(\begin{array}{ccc}
-6 & -5 & -1 \\
& -7 & 0 \\
& & 4
\end{array}\right) \times 10^{-5}
$$

and

$$
\sigma=\left(\begin{array}{ccc}
-20 & -6 & 2 \\
& -20 & 3 \\
& & 0
\end{array}\right) \mathrm{MPa}
$$

The computation was based on 18 lattice planes $\{551\}$ and $\{711\}$ corresponding to high Bragg angle $\left(76^{\circ}\right)$.

Despite very different spatial resolutions, the two methods (Kossel microdiffraction and XRD) exhibit similar strain resolutions. The gaps between corresponding stress components are lower than $10 \mathrm{MPa}$. Both methods lead to normal stress components slightly in compression and shear components close to zero. The results are consistent with expectations for a macroscopically stress-free sample. On the basis of the above, the strain resolution obtained from very sharp experimental Kossel patterns and a large number of conics can reach $1 \times 10^{-4}$.

\section{Stresses in a uniaxially strained nickel-based superalloy}

Finally, the Kossel technique was tested by measurement of nonzero stresses in single crystals of a nickelbased superalloy with a high yield stress. Strains in these crystals were determined during uniaxial straining. With this approach, the internal stresses measured by the Kossel technique can be referred to the known values of the applied stress. Results obtained from Kossel patterns recorded using SEM were also compared with stresses acquired by classical XRD measurements and with results of measurements by synchrotron-based Kossel diffraction. Although the experiments were carried out on different occasions, on different specimens and with different loads, the material was always the same, and also the same tensile device was used in all three cases.

The alloy was provided by ONERA. Its chemical composition (in weight \%) was $\mathrm{Ni}$ base, Cr 8\%, Co 5\%, W 8\%, Mo 2\%, Al 5\%, Ti $1.5 \%$ and $\mathrm{Ta} 6 \%$. The material consisted of two coherent phases: $\mathrm{Ni} \gamma$ matrix and $\left[(\mathrm{Ni}, \mathrm{Co}, \mathrm{Cr})_{3}-\right.$ 
Table 2

Stress tensors obtained by Kossel microdiffraction in a scanning electron microscope, by Kossel diffraction using synchrotron radiation and by single-crystal XRD during in situ uniaxial tensile straining.

The component $\sigma_{11}$ is along the tensile direction. All values are in MPa.

Kossel.

\begin{tabular}{llccc}
\hline Applied stress & 0 & & 400 & 680 \\
\hline Stress tensor & $\left(\begin{array}{cccc}-95 & 5 & 0 \\
& -15 & 0 \\
& & 0\end{array}\right)\left(\begin{array}{lll}360 & -55 & 0 \\
& -45 & 0 \\
& & 0\end{array}\right)\left(\begin{array}{lll}635 & -25 & 0 \\
& -35 & 0 \\
& & 0\end{array}\right)$
\end{tabular}

Synchrotron.

\begin{tabular}{|c|c|c|c|c|c|c|c|c|c|}
\hline Applied stress & 0 & & & 400 & & & 750 & & \\
\hline Stress tensor & $(1$ & $\begin{array}{c}-105 \\
30\end{array}$ & $\begin{array}{l}0 \\
0 \\
0\end{array}$ & $\left(^{365}\right.$ & $\begin{array}{l}-25 \\
-30\end{array}$ & $\left.\begin{array}{l}0 \\
0 \\
0\end{array}\right)$ & (655 & $\begin{array}{c}-15 \\
80\end{array}$ & $\left.\begin{array}{l}0 \\
0 \\
0\end{array}\right)$ \\
\hline
\end{tabular}

XRD.

Applied stress 0 650

Stress tensor

$$
\left(\begin{array}{ccc}
0 & 45 & -10 \\
& -105 & 10 \\
& & 0
\end{array}\right)
$$$$
\left(\begin{array}{ccc}
585 & 40 & -20 \\
& -40 & 10 \\
& & 75
\end{array}\right)
$$

(Al,Ti,Ta)] cubic $\gamma^{\prime}$ precipitates (Durand-Charre, 1997) (see Fig. 6). The crystal orientation in the specimen was near $\{011\}\langle 611\rangle$. Dog-bone samples were machined and the gauge areas were then polished (mechanically and electrolytically). The strain-stress behavior of the material was investigated by a conventional (macro) tensile test. The deformation was elastic at the macroscale up to about $900 \mathrm{MPa}$, and therefore it was assumed to be linearly elastic at the microscale at the level of $750 \mathrm{MPa}$ (the highest stress applied in subsequent tests). The elastic stiffness tensor used for stress calculation was determined from the temperature-dependent law given by Gaubert (2009); components of this tensor are listed in Table 1. The reference lattice parameter was $a=3.595 \AA$.

Using SEM, three different locations, several hundreds of micrometres apart, were randomly selected in the center of the specimen. Kossel patterns were recorded at the initial state (no loading) and for two uniaxial applied stresses (400 and $680 \mathrm{MPa}$ ). In all cases, the patterns were more diffuse than those of Ge, with blurred and relatively wide Kossel lines. An example experimental pattern is shown in Fig. 7(a). Only ten conics per pattern were used. As Fig. 4(b) indicates, fitting all strain components to ten lines would inevitably lead to considerable errors. Therefore, following Bauch et al. (2000), to reduce the number of free fitting parameters, a biaxial stress state was assumed. Two out of the ten conics used for the refinement of lattice parameters were bright-dark. Fig. 7(b) with geometrically simulated lines superimposed on the experimental pattern shows the presence of some extra conics.
Table 3

Values of $\sigma_{11}$ (in $\mathrm{MPa}$ ) obtained by Kossel microdiffraction in a scanning electron microscope for three locations and two applied stresses (400 and $680 \mathrm{MPa}$ ), and the standard deviations per loading.

\begin{tabular}{lll}
\hline Applied stress & 400 & 680 \\
\hline$\sigma_{11}$ for spot 1 & 435 & 700 \\
$\sigma_{11}$ for spot 2 & 340 & 585 \\
$\sigma_{11}$ for spot 3 & 305 & 615 \\
Average (standard deviation) & $360(65)$ & $633(60)$ \\
\hline
\end{tabular}

They correspond to $\mathrm{Ni} K \beta$ and to radiation emitted by other elements $(\mathrm{Cr} K \alpha$ and $\mathrm{Co} K \alpha)$. As the wavelengths are different from that of $\mathrm{Ni} K \alpha$, these additional reflections do not affect the analysis of the pattern.

Kossel patterns were also obtained at the European Synchrotron Radiation Facility in Grenoble (ESRF, ID13 beamline) using a monochromatic synchrotron beam. The beam size was about $3 \mu \mathrm{m}$. The $9.4 \mathrm{keV}$ photon energy was chosen to be just above the $K$ absorption edge of nickel. This technique has the advantage of working in air so it is easier to put a detector and a tensile device in a desired position, but the exposure times had to be multiplied by a factor of five compared to the times of the SEM measurements. Kossel patterns were recorded at the initial state and for two uniaxial applied stresses: 400 and $750 \mathrm{MPa}$. A single location was analyzed. Also in this case, a biaxial stress state was assumed.

In the case of the XRD-based measurements, the mean stress state was obtained from a volume much larger than that of Kossel microdiffraction. The analyzed surface area was about $1 \mathrm{~mm}^{2}$. The stresses were measured before loading and for the applied stress of $650 \mathrm{MPa}$.

The stress results obtained by the three methods are collected in Table 2. They are given in the sample coordinate system with $\sigma_{11}$ corresponding to the loading direction. In the case of SEM-based data, the final stress tensors were obtained by averaging the three different measurements. The $\sigma_{11}$

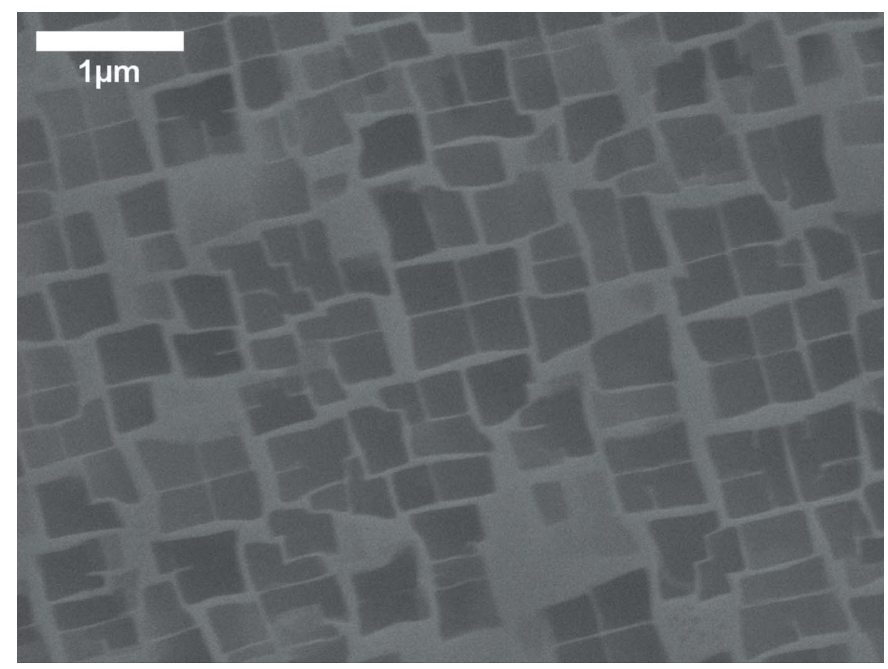

Figure 6

Microstructure of the Ni superalloy, showing a $\gamma$ matrix and cubic $\gamma^{\prime}$ precipitates. 
components were about $40 \mathrm{MPa}$ lower than the applied stress, and $\sigma_{22}$ and $\sigma_{12}$ were slightly in compression. Besides that, $\sigma_{11}$ had significant dispersion of about $60 \mathrm{MPa}$ (Table 3). As for the results of the experiments at ESRF, the local stress tensor for the applied stress of $400 \mathrm{MPa}$ was very close to that obtained by SEM. However, for the $750 \mathrm{MPa}$ state, a larger gap (110 MPa) between $\sigma_{11}$ and the applied stress was observed. The XRD results are similar to those of Kossel microdiffraction: in the former case, $\sigma_{11}$ was slightly lower than the applied stress (about 10\% difference) and the other components were negligible compared to $\sigma_{11}$. From the shear stresses and the dispersion (Table 2), the stress resolution of the Kossel microdiffraction in SEM can reasonably be estimated to be about $60 \mathrm{MPa}$. For the investigated alloy, it is equivalent to a strain resolution of about $3 \times 10^{-4}$.

Besides the imprecision of the used methods, there are other possible causes of deviations between the applied

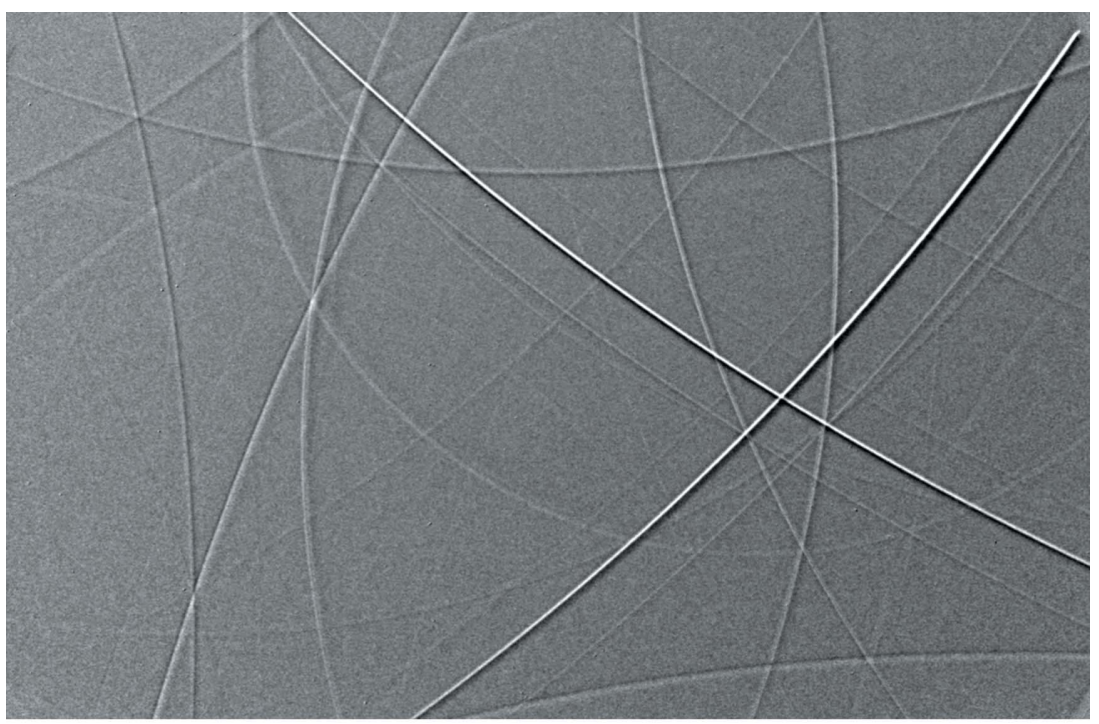

$(a)$

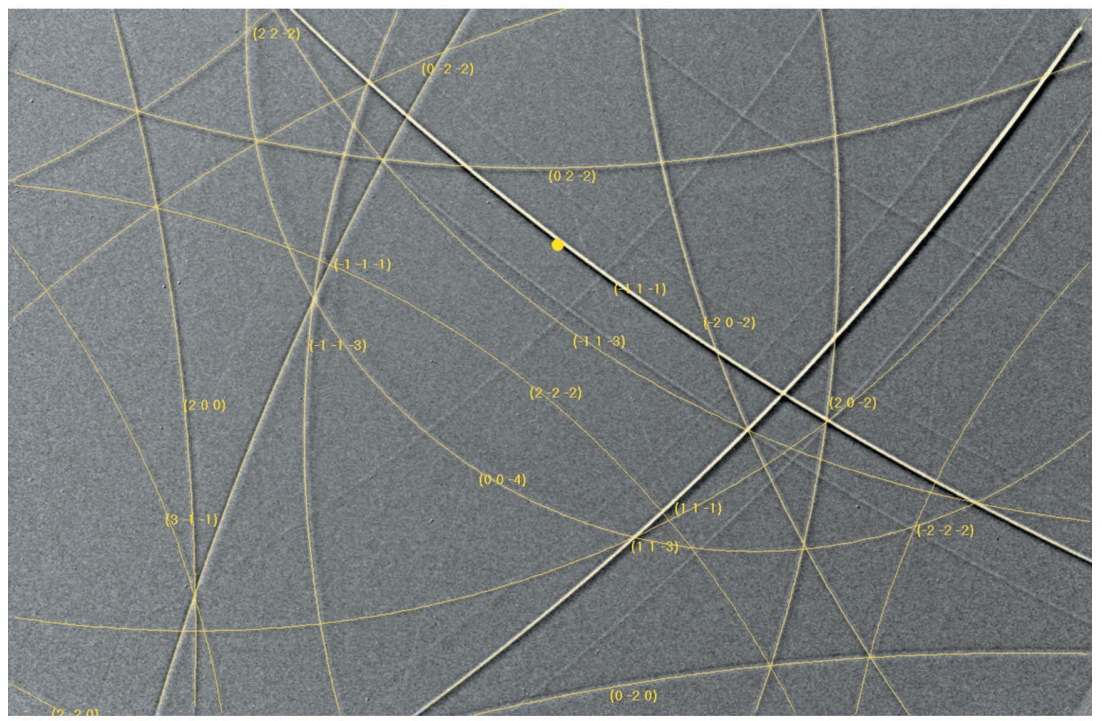

(b)

Figure 7

(a) Experimental Kossel pattern obtained from the Ni superalloy. (b) Geometric simulation superimposed on the experimental pattern. stresses and the internal stress along the tensile direction. In the loaded states, the gaps can be partly explained by the experiment duration, which could allow for a relaxation of the stress imposed by the tensile device. (The recording of Kossel patterns in the scanning electron microscope took about three minutes per pattern plus the time needed to adjust the specimen and the electron beam. The XRD experiment took several hours as the crystal was oriented before each measurement of interplanar spacing.) The deviations could also be linked to stress localization. Indeed, the SEM measurements showed that $\sigma_{11}$ depends on the location. Some deviations may be due to the composite microstructure of the material and differences in the volumes analyzed in SEM-, synchrotron- and XRD-based measurements, as the stress values are averages over $\gamma$ and $\gamma^{\prime}$ phases having different mechanical properties. Also, heterogeneities caused by surface preparation could contribute to the deviations. In fact, residual stresses obtained in the initial (no loading) states were significant.

\section{Conclusions}

A Kossel microdiffraction setup has been assembled in a scanning electron microscope for strain and stress analysis at the micrometre scale. The strain resolution of Kossel microdiffraction was estimated using simulated diffraction patterns. The effect of the number of conics selected for the refinement was investigated. The test provided the minimum number of conics that must be marked on the experimental pattern to achieve a given strain accuracy. For the considered Ni alloy, if the full strain tensor is calculated, in order to reach a precision of $2 \times 10^{-4}$, at least 11 Kossel conics need to be taken into account. On the other hand, the achievable strain resolution estimated from a very sharp experimental pattern was found to be about $1 \times 10^{-4}$. The Kossel technique was finally tested for determination of nonzero stresses in single crystals of a nickelbased superalloy during uniaxial straining. Stress results obtained by Kossel microdiffraction (in SEM and with synchrotron radiation) were compared with those collected from X-ray diffraction. For this material, the strain and the stress resolutions were estimated to be about $3 \times 10^{-4}$ and $60 \mathrm{MPa}$, respectively. The strain resolution of the proposed approach is constrained by the crystal structure, which determines visible reflections and their Bragg angles, and - intrinsically - by the use of a simple geometric description of diffraction lines in combination with empirical rules of matching the geometric lines to experimental lines (of finite thickness and complicated profiles). 
The strain resolution obtained for the $\mathrm{Ni}$ superalloy $(3 \times$ $10^{-4}$ ) demonstrates that the Kossel microdiffraction technique can be effectively used for practical studies of moderate local elastic stresses in polycrystalline samples with grain dimensions of a few micrometres or more.

The authors wish to thank P. Caron from ONERA for providing the superalloy single crystal and M. Burghammer from ESRF for invaluable assistance during the synchrotronbased experiments. The work of the French authors was partially funded by the French National Agency (ANR) within the SAKE project (ANR PNANO-07-029). The work of AM was supported by the National Science Center based on decision DEC-2012/06/M/ST8/00449.

\section{References}

Barbe, F., Forest, S. \& Cailletaud, G. (2001). Int. J. Plast. 17, 537-563.

Barna, S. L., Tate, M. W., Gruner, S. M. \& Eikenberry, E. F. (1999). Rev. Sci. Instrum. 70, 2927-2934.

Bauch, J., Brechbühl, J. \& Ullrich, H. J. (2000). Adv. X-ray Anal. 42, 471-481.

Böhling, M. \& Bauch, J. (2007). Cryst. Res. Technol. 42, 905-913.

Bouscaud, D., Pesci, R., Berveiller, S. \& Patoor, E. (2012). Ultramicroscopy, 115, 115-119.

Clément, L., Pantel, R., Kwakman, L. F. T. \& Rouvière, J. L. (2004). Appl. Phys. Lett. 85, 651-653.

Crostack, H. A., Reimers, W. \& Eckold, G. (1987). Residual Stresses in Science and Technology, edited by E. Macherauch \& V. Hauk, pp. 281-294. Oberursel: DGM Informationsgesellschaft Verlag.
Cunningham, B. \& Ashbee, K. (1990). Acta Metall. Mater. 38, 25612565.

De Wolf, I., Ignat, M., Pozza, G., Maniguet, L. \& Maes, H. E. (1999). J. Appl. Phys. 82, 6477-6485.

Dingley, D. J. (1975). Scanning Electron Microscopy. Proceedings of the 8th Annual SEM Symposium, edited by O. Johari \& I. Corvin, pp. 173-180. Chicago: IIT Research Institute.

Durand-Charre, M. (1997). The Microstructure of Superalloys. London: Gordon and Breach.

Gaubert, A. (2009). PhD thesis, École Nationale Supérieure des Mines de Paris, France.

Hauk, V. (1997). Structural and Residual Stress Analysis by Nondestructive Methods. Amsterdam: Elsevier.

Kossel, W., Loeck, V. \& Voges, H. (1935). Z. Phys. 94, 139-144.

Krämer, S., Mayer, J., Witt, C., Weickenmeier, A. \& Rühle, M. (2000). Ultramicroscopy, 81, 245-262.

Lamaze, J. P. \& Despujols, J. (1976). J. Phys. E Sci. Instrum. 9, 41-43.

Langer, E. \& Daebritz, S. (2010). IOP Conf. Ser. Mater. Sci. Eng. 7, 012015.

Lider, V. V. (2011). Crystallogr. Rep. 56, 169-189.

Morawiec, A. (1999). J. Appl. Cryst. 32, 788-798.

Morawiec, A. (2007). Ultramicroscopy, 107, 390-395.

Morawiec, A., Pesci, R. \& Lecomte, J. S. (2008). Ceram. Trans. 201, 163-169.

Ortner, B. (2005). J. Appl. Cryst. 38, 678-684.

Pesci, R., Inal, K., Berveiller, S., Patoor, E., Lecomte, J. S. \& Eberhardt, A. (2006). Mater. Sci. Forum, 524, 109-114.

Ruud, C. (1982). NDT Int. 15, 15-23.

Tamura, N., Padmore, H. \& Patel, J. (2005). Mater. Sci. Eng. 399, 92 98.

Tixier, R. \& Waché, C. (1970). J. Appl. Cryst. 3, 466-485.

Wilkinson, A. J., Meaden, G. \& Dingley, D. J. (2009). Superlattice Microstruct. 45, 285-294. 